\title{
Modeling and Management of Bridge Structures Using Unmanned Aerial Vehicle in Korea
}

\author{
Keun Wang Lee ${ }^{1}$ and Joon Kyu Park ${ }^{2 *}$ \\ ${ }^{1}$ Department of Multimedia Science, Chungwoon University, 113, Sukgol-ro, Nam-gu, Incheon 22100, Korea \\ ${ }^{2}$ Department of Civil Engineering, Seoil University, 28, Yongmasan-ro 90-gil, Jungnang-gu, Seoul 02192, Korea
}

(Received June 2, 2019; accepted August 9, 2019)

Keywords: 3D modeling, bridge structure, geospatial information, management, modeling, terrestrial LiDAR, UAV

All facilities become obsolete over time and require continuous maintenance and monitoring for safety. Bridges should be maintained to preserve their function during their life time and regularly inspected and repaired to maintain the convenience and safety of users, and their damaged parts should be restored or improved. All these activities are necessary for reinforcement. To maintain bridges, it takes a long time to accumulate geospatial information data because the drawing of the site surveyed using manpower and the creation of a management register depend on manual operation. In this study, 3D modeling of a bridge using an unmanned aerial vehicle (UAV; hereafter, UAV modeling) was performed to generate data for bridge maintenance, and the accuracy and usability of UAV modeling results were evaluated by comparing them with those of terrestrial light detection and ranging (LiDAR) results. The UAV modeling results showed an accuracy of less than $0.12 \mathrm{~m}$, which suggests the usefulness of UAV modeling for bridge maintenance. The UAV modeling results can also be used for the maintenance of bridges by providing image information to determine the condition of the bridge surface. If the facilities are maintained using the UAV, the efficiency of work related to the management of road facilities is expected to improve.

\section{Introduction}

As society develops, the desire to pursue a higher standard of living is increasing, and accordingly, many urban facilities are being built. ${ }^{(1,2)}$ In particular, bridges are used for various purposes such as accident prevention, disaster prevention, and driver convenience; therefore their proper maintenance is required. ${ }^{(3)}$ Bridges should be maintained to preserve their function during their life time and regularly inspected and repaired to maintain the convenience and safety of users, and their damaged parts should be restored or improved. All these activities are necessary for reinforcement. ${ }^{(4)}$ To maintain bridges, it takes a long time to construct data because the drawing of the site surveyed using manpower and the creation of the management register depend on manual operation. ${ }^{(5,6)}$ In this study, modeling for bridge maintenance using

*Corresponding author: e-mail: jkpark@seoil.ac.kr https://doi.org/10.18494/SAM.2019.2467 
terrestrial light detection and ranging (LiDAR) data and unmanned aerial vehicle (UAV) data was performed. UAVs are cheap and easy to operate, especially for quick access to desired areas. Owing to the rapid transmission of the images of a target area acquired by UAVs, they are widely used in fields such as military, communication, entertainment, and agriculture. ${ }^{(7,8)}$ Unlike conventional aerial and satellite images, which capture images in accordance with a predetermined cycle and schedule, a UAV is capable of responding instantaneously under conditions that change rapidly owing to, for example, a disaster, and providing much information. Moreover, it can be used in the field of maintenance. ${ }^{(9,10)}$ In recent years, there have been many studies using UAVs such as GNSS / INS to improve the UAV position and attitude information performance and digital camera performance. ${ }^{(11,12)}$ In this study, the 3D modeling of bridge using an UAV (hereafter, UAV modeling) was performed to generate data for bridge maintenance, and the accuracy and usability of UAV data were evaluated by comparing them with terrestrial LiDAR data. Figure 1 shows the study flow of this study.

\section{Data Acquisition and Processing}

In this study, a bridge of the Han River was selected as the study site for the modeling for bridge maintenance. The length of the bridge is about 100 meters, and data were acquired using terrestrial LiDAR and an UAV. Figure 2 shows the study area. Figures 3 and 4 show the terrestrial LiDAR and UAV used for data acquisition, respectively.

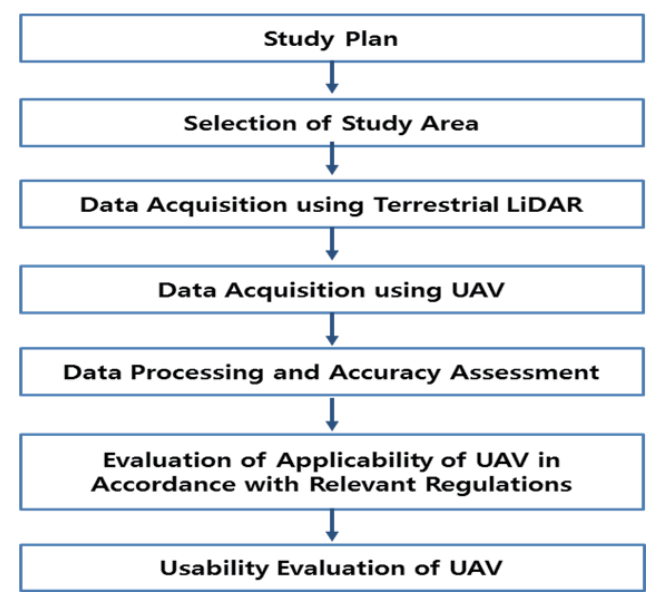

Fig. 1. Study flow.

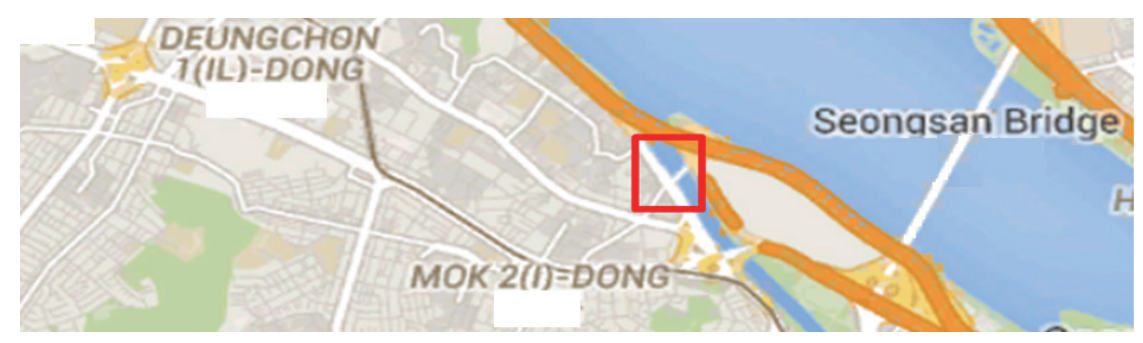

Fig. 2. (Color online) Study area. 


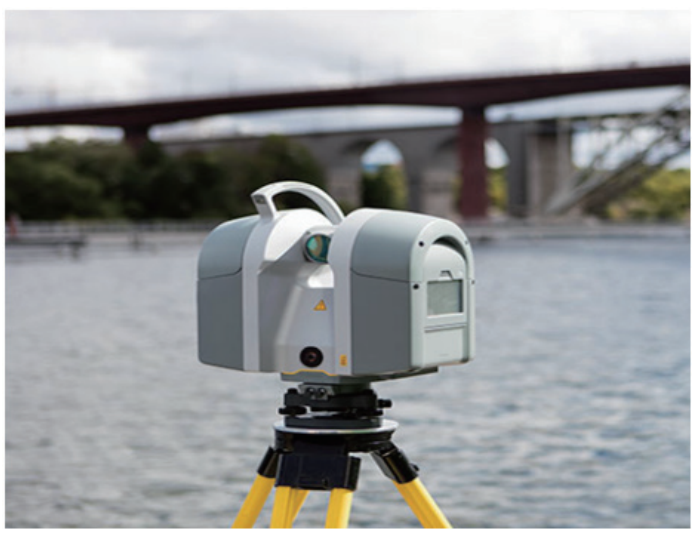

Fig. 3. (Color online) Terrestrial LiDAR. ${ }^{(13)}$

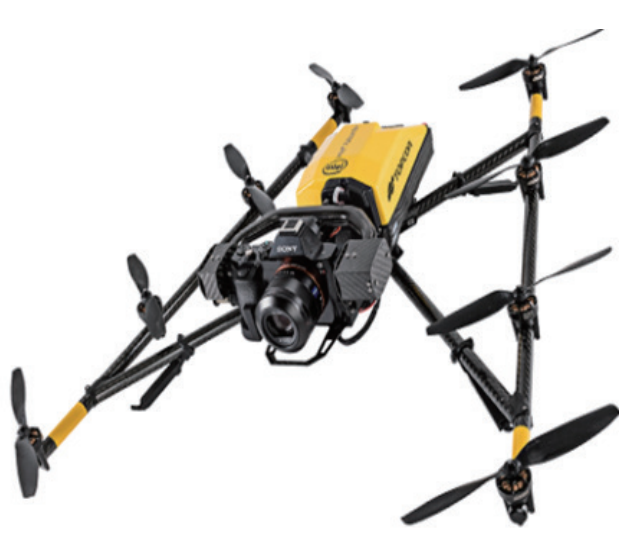

Fig. 4. (Color online) $\mathrm{UAV}^{(14)}$

Table 1

Coordinates of the control points.

\begin{tabular}{lccc}
\hline \multirow{2}{*}{ Point } & \multicolumn{3}{c}{ Coordinates } \\
\cline { 2 - 4 } & $X(\mathrm{~m})$ & $Y(\mathrm{~m})$ & $H(\mathrm{~m})$ \\
\hline BTP01 & 550187.0054 & 189226.0484 & 14.9153 \\
BTP02 & 550225.9999 & 189257.4571 & 14.5334 \\
BTP03 & 550184.2076 & 189229.4596 & 14.1113 \\
BTP04 & 550223.1913 & 189260.8533 & 13.8554 \\
BTP05 & 550255.4719 & 189284.0437 & 13.9018 \\
BTP06 & 550184.2063 & 189229.5049 & 4.9963 \\
BTP07 & 550180.1641 & 189233.3103 & 13.1488 \\
\hline
\end{tabular}

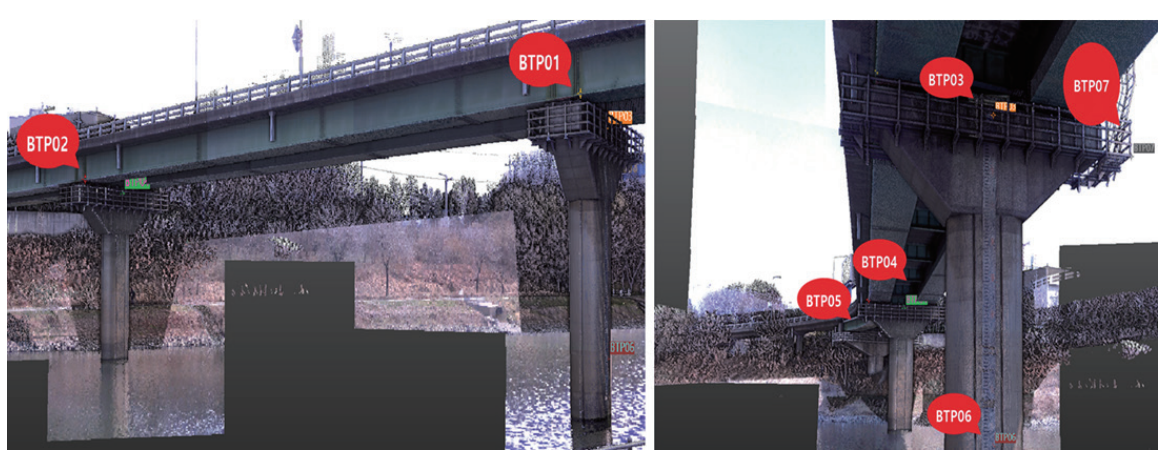

Fig. 5. (Color online) Positions of control points.

First, the control points were surveyed for the processing of the acquired data. Seven control points were selected. Table 1 shows the coordinates of the control points, and Fig. 5 shows the positions of the control points.

Data on the bridge in the study area were obtained by moving the terrestrial LiDAR three times along a length of about $100 \mathrm{~m}$. Data were acquired during automatic and manual flights of the UAV. Since no GNSS signals are received at the lower part of the bridge, manual flight is performed. About 1000 images were acquired with the UAV. Figure 6 shows some of the images acquired with the UAV. 

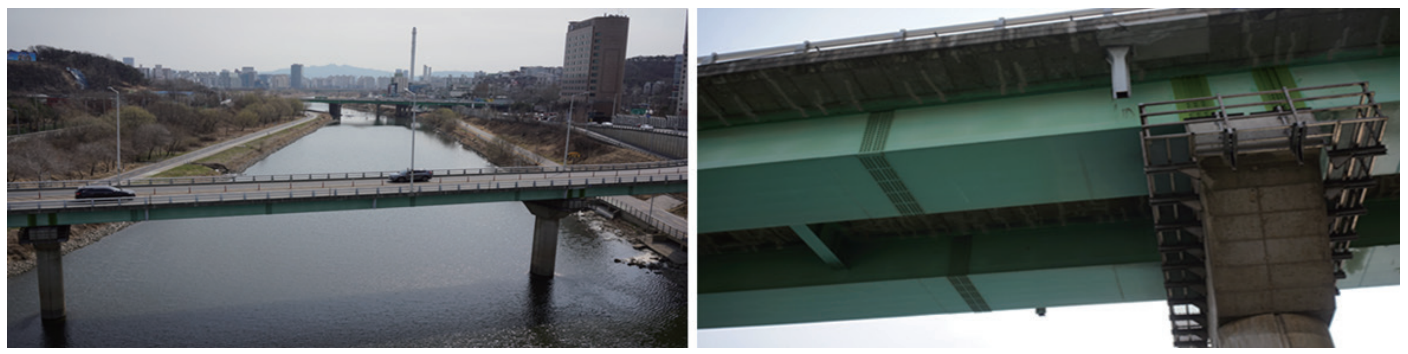

Fig. 6. (Color online) Images acquired with UAV.

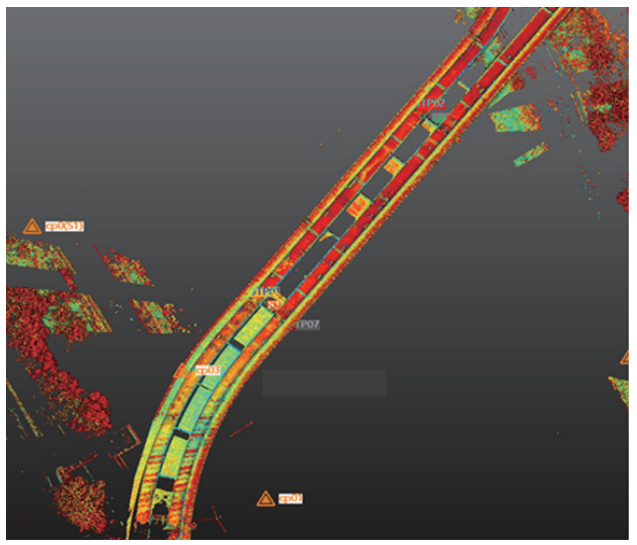

Fig. 7. (Color online) Point cloud.

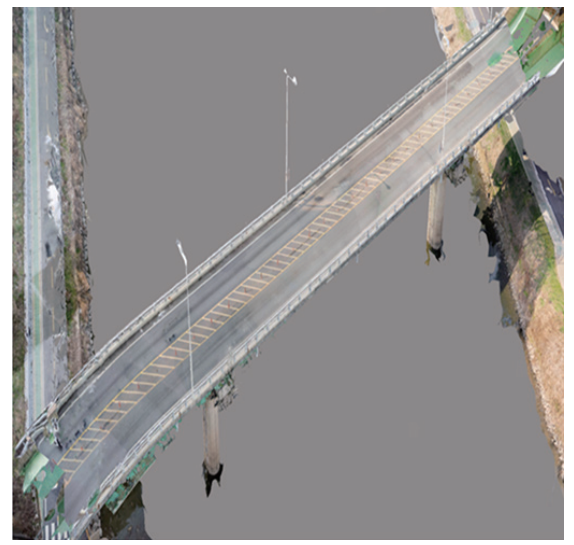

Fig. 8. (Color online) UAV modeling result.

Terrestrial LiDAR data, which were used for comparison to determine the accuracy of the UAV data, were processed using Trimble Real Works (TRW) software for "registration" and "georeferencing". UAV data were processed using UAS Master Software. Tie point extraction, reference point observation, and 3D modeling data generation were carried out for 3D data generation and modeling. Through data processing, a terrestrial LiDAR point cloud was created for the study site, and a modeling result of the bridge obtained using the UAV was generated. Figure 7 shows a terrestrial LiDAR point cloud, and Fig. 8 shows the result of UAV modeling.

\section{Analysis of UAV Results}

As shown in Fig. 6, UAV modeling provides excellent and clear images because it can display the three-dimensional shape and color of the bridge. The terrestrial LiDAR pointcloud does not have a color value because no image was acquired. However, it can be used to evaluate the accuracy of the UAV modeling results because it shows the exact shape of the bridge. A total of 10 checkpoints were set and compared with the UAV modeling results. Table 2 shows the accuracy evaluation of the UAV modeling results.

The accuracy of the UAV modeling results is within $0.12 \mathrm{~m}$, as shown in Table 2. These results show that the UAV can be used for the modeling of bridges. On the other hand, the UAV modeling results for the bridge was found to be useful for its maintenance because UAV modeling provides high-resolution images. It is possible to inspect facilities such as bolts and 
Table 2

Accuracy evaluation of UAV modeling results.

\begin{tabular}{|c|c|c|c|c|c|c|c|}
\hline \multirow{2}{*}{ Point } & \multicolumn{3}{|c|}{ Deviation } & \multirow{2}{*}{ Point } & \multicolumn{3}{|c|}{ Deviation } \\
\hline & $d X(\mathrm{~m})$ & $d Y(\mathrm{~m})$ & $d H(\mathrm{~m})$ & & $d X(\mathrm{~m})$ & $d Y(\mathrm{~m})$ & $d H(\mathrm{~m})$ \\
\hline Checkpoint1 & 0.07 & 0.05 & 0.08 & Checkpoint6 & 0.07 & 0.05 & 0.06 \\
\hline Checkpoint2 & 0.02 & 0.08 & 0.12 & Checkpoint7 & 0.09 & 0.09 & 0.09 \\
\hline Checkpoint3 & 0.03 & 0.07 & 0.07 & Checkpoint8 & 0.08 & 0.08 & 0.08 \\
\hline Checkpoint4 & 0.09 & 0.05 & 0.05 & Checkpoint9 & 0.05 & 0.07 & 0.04 \\
\hline Checkpoint5 & 0.08 & 0.04 & 0.11 & Checkpoint10 & 0.05 & 0.04 & 0.12 \\
\hline
\end{tabular}
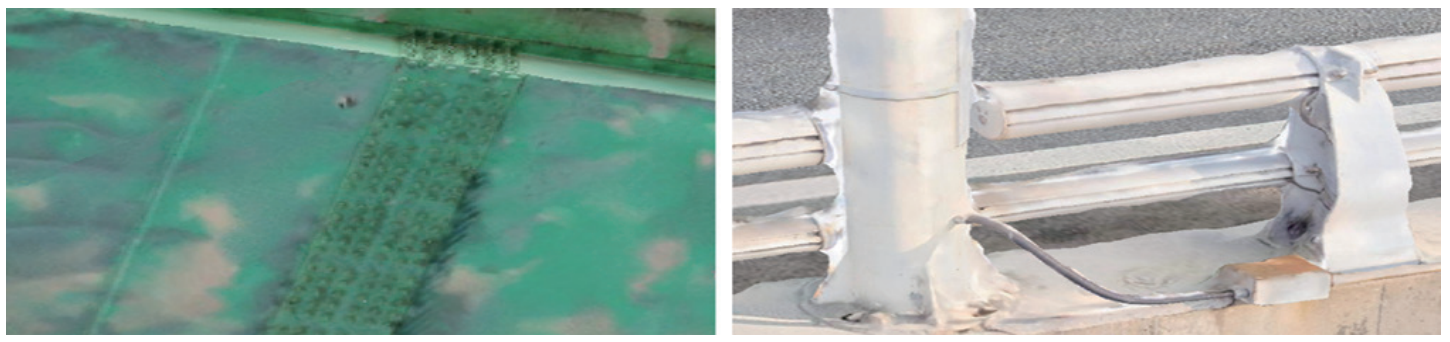

Fig. 9. (Color online) Effect of water reflection and distortion of the modeling result.
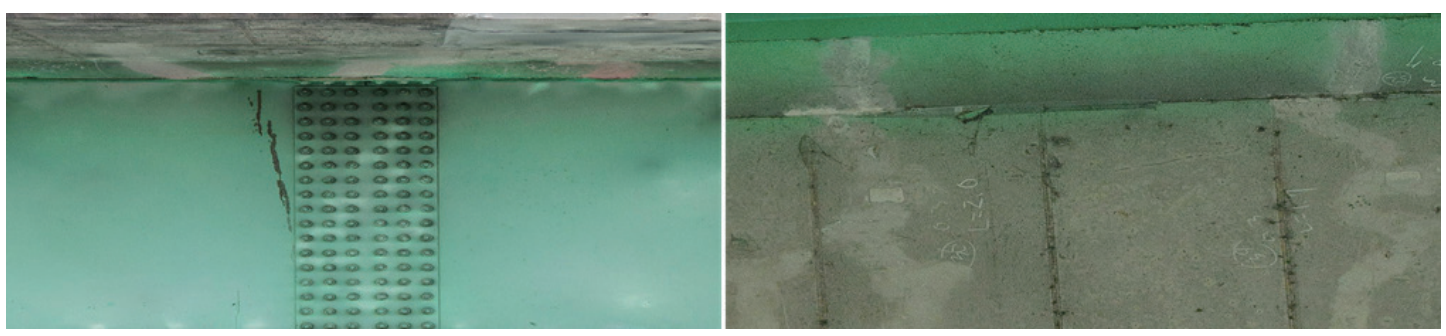

Fig. 10. (Color online) Close-up of UAV modeling result.

signs of bridges and to monitor the lower part of bridges, which is difficult for people to access. The image of the lower part of the bridge is slightly affected by the reflection of water, but it is sufficiently useful for detecting factors such as the corrosion of bridge substructures. Moreover, it is expected that this can be improved by correcting raw images. Figure 9 shows the effect of water reflection and distortion on the modeling result, and Fig. 10 shows a close-up of the UAV modeling result and the possibility of inspection using a UAV.

As shown in Fig. 8, the UAV modeling result can be used for the maintenance of bridges by providing image information to determine the condition of the bridge surface. If the facilities are maintained using an UAV, the efficiency of work related to the management of road facilities is expected to improve.

\section{Analysis of UAV utilization for Facility Maintenance}

The UAV modeling results of facilities show very clear images as shown in Sect. 3 and can be used for maintenance work. In this study, the utilization of the UAV was analyzed on the basis of the maintenance regulations of facilities. There are special laws governing the safety 
and maintenance of facilities in Korea. In these laws, facilities are classified into classes 1, 2, and 3. Table 3 shows the definitions of facilities by class. ${ }^{(15)}$

Safety checks for class 1, 2, and 3 facilities consist of periodic and precise safety inspections. Regular safety checks involve the survey of facilities, and precise safety checks involve the external inspection of the main components of facilities using measurement equipment. As shown in Fig. 8, the resolution of the image acquired by the UAV is high enough to be used for inspecting the exterior of a facility. To determine the usefulness of UAVs for the precise safety inspection of facilities, the important parts of a structure defined by related laws and their defects were identified, and the usefulness of the UAVs was evaluated. Table 4 shows the critical parts of the structures and the deficiencies that should be checked as specified by the laws.

The UAV was found to be applicable in the safety inspections and maintenance of bridges, river facilities, dam facilities, buildings, and harbors. In the case of tunnel or building interiors, it is possible to inspect for their safety and maintenance using a UAV if space for UAV flight

Table 3

Accuracy evaluation of UAV modeling results.

\begin{tabular}{lc}
\hline Class & Definition \\
\hline 1 & Bridges over 500 meter; tunnels over 1000 meter; buildings over 21 stories, etc. \\
2 & Bridges over 100 meter; tunnels, harbors, dams, buildings over 16 floors, etc. (excluding class 1) \\
3 & Bridges less than 100 meter; tunnels; harbors, dams, buildings over 16 floors, etc. (excluding class 2) \\
\hline
\end{tabular}

Table 4

Critical parts of structures and deficiencies that should be checked as specified by laws.

\begin{tabular}{|c|c|c|}
\hline Structure & Deficiency & UAV application \\
\hline \multirow{7}{*}{ Bridge } & Lack of rebar in major structural parts & $\times$ \\
\hline & Deepening of mold crack & ० \\
\hline & Severe material separation of reinforced concrete members & ० \\
\hline & Cracks and severe deformation of member connection plates & ० \\
\hline & Welding defect of steel weld & ○ \\
\hline & Damage to cable member or tension member & ○ \\
\hline & Cracks in alternation and pier & 0 \\
\hline \multirow{2}{*}{ Tunnel } & Deepening and elimination of wall cracks & $x$ \\
\hline & Leakage and deformation & $x$ \\
\hline River facilities & Poor operation of water gate & o \\
\hline \multirow{3}{*}{ Dam facilities } & Leakage and deformation & 0 \\
\hline & Poor operation of water gate & $\circ$ \\
\hline & Poor bonding of piping & $x$ \\
\hline \multirow{2}{*}{ Water supply facilities } & Damage, deformation, and corrosion of pipeline & $x$ \\
\hline & Connecting part of prefabricated structure & $x$ \\
\hline \multirow{3}{*}{ Building } & Excessive deformation and cracking in major structural members & o \\
\hline & Ground subsidence and active cracking & ○ \\
\hline & Leakage and corrosion & ○ \\
\hline \multirow{4}{*}{ Harbor } & Corrosive aging of door-to-door operation facilities & ० \\
\hline & $\begin{array}{l}\text { Deterioration of corrosion in aciduct facilities of capsular filled with } \\
\text { water and drainage }\end{array}$ & $x$ \\
\hline & Structural damage & o \\
\hline & Normal displacement and settlement of quay wall & $\times$ \\
\hline
\end{tabular}


and illumination for image acquisition are available. The UAV can access areas that are inaccessible to humans and can document the results of monitoring through photography or video imaging. In addition, UAV modeling results can be used for the maintenance of facilities because deriving 3D coordinate results and quantitative analysis are possible.

\section{Conclusions}

In this study, the 3D modeling of a bridge using a UAV was performed to generate data for bridge maintenance, and the accuracy and usability of this method were evaluated by comparing them with those terrestrial LiDAR. Data on the bridge of the study area we acquired using terrestrial LiDAR and a UAV, and the point cloud and modeling results of the bridge were obtained through data processing. UAV modeling results were compared with terrestrial LiDAR results to evaluate their accuracy. The UAV modeling results showed an accuracy of less than $0.12 \mathrm{~m}$, which suggests the usefulness of UAV modeling for bridge maintenance. On the basis of the laws on facility maintenance, the usefulness of UAV modeling was analyzed, and results suggest that the UAV modeling can be used for the maintenance of class 1,2 , and 3 facilities. UAV modeling results can also be used for the maintenance of bridges by providing image information to determine the condition of the bridge surface. If the facilities are maintained using the UAV, the efficiency of work related to the management of road facilities is expected to improve.

\section{Acknowledgments}

This research was supported by the Basic Science Research Program through the National Research Foundation of Korea (NRF) funded by the Ministry of Science and ICT (No. NRF2018R1C1B6004021).

\section{References}

1 D. Y. Han, J. B. Park, and J. W. Huh: J. Korean Soc. Surv. Geod. Photogramm. Cartography 36 (2018) 451. https://doi.org/10.7848/ksgpc.2018.36.6.451

2 M. C. Kim and H. J. Yoon: J. Korean Academia-Industrial Cooperation Soc. 19 (2018) 97. https://doi. org/10.5762/KAIS.2018.19.2.97

3 L. Matikainen, M. Lehtomäki, E. Ahokas, J. Hyyppä, M. Karjalainen, A. Jaakkola, A. Kukko, and T. Heinonen: ISPRS J. Photogramm. Remote Sens. 119 (2016) 10. https://doi.org/10.1016/j.isprsjprs.2016.04.011

4 J. H. Oh, Y. J. Jang, and C. N. Lee: J. Korean Soc. Surv. Geod. Photogramm. Cartography 36 (2018) 565. https://doi.org/10.7848/ksgpc.2018.36.6.565

5 P. Martinez-Carricondo, F. Aguera-Vega, F. CarvajalRamirez, F. Mesas-Carrascosa, A. Garcia-Ferrer, and F. Perez-Porras: Int. J. Appl. Earth Obs. Geoinf. 72 (2018) 1. https://doi.org/10.1016/j.jag.2018.05.015

6 J. K. Park and D. Y. Um: J. Korean Academia-Industrial Cooperation Soc. 19 (2018) 155. https://doi. org/10.5762/KAIS.2018.19.3.155

7 S. D. Jawak, S. N. Panditrao, and A. J. Luis: Spatial Inf. Res. 26 (2018) 483. https://doi.org/10.1007/s41324018-0191-1

8 W. J. Kim, C. J. Kim, Y. J. Cho, J. S. Kim, H. J. Kim, D. H. Lee, O. Y. Lee, and J. P. Meng: J. Korean Soc. Surv. Geod. Photogramm. Cartography 35 (2017) 553. https://doi.org/10.7848/ksgpc.2017.35.6.553

9 E. I. Jeon, S. J. Yu, H. W. Seok, S. J. Kang, K. Y. Lee, and O. S. Kwon: Spatial Inf. Res. 25 (2017) 701. https:// doi.org/10.1007/s41324-017-0137-z 
10 J. K. Park and K. Y. Jung: J. Korean Academia-Industrial Cooperation Soc. 19 (2018) 602. https://doi. org/10.5762/KAIS.2018.19.2.602

11 M. K. Chung, C. J. Kim, K. H. Choi D. K. Chung, and Y. I. Kim: J. Korean Soc. Surv. Geod. Photogramm. Cartography 35 (2017) 91. https://doi.org/10.7848/ksgpc.2017.35.2.91

12 J. W. Lee and S. M. Sung: J. Korean Academia-Industrial Cooperation Soc. 19 (2018) 63. https://doi. org/10.5762/KAIS.2018.19.4.63

13 Trimble Navigation Ltd. (2019): http://www.trimble.com.

14 Intel Corporation (2019): https://www.intel.com/content/www/us/en/products/drones/falcon-8.html (accessed April 2019).

15 Ministry of Government Legislation: http://www.law.go.kr/ (accessed April 2019).

\section{About the Authors}

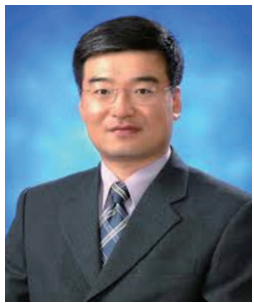

Keun Wang Lee is a professor in the Department of Multimedia Science at Chungwoon University. He received his B.S. degree in electronic computing from Hanbat University in 1993 and his M.S. and Ph.D. degrees in computer science from Soongsil University, Korea in 1996 and 2000, respectively. His research interests are in the areas of multimedia communication and multimedia application. (kwlee@chungwoon.ac.kr)

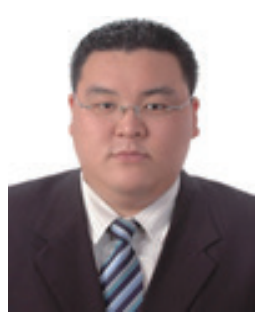

Joon Kyu Park is currently a professor in the Department of Civil Engineering at Seoil University. He received his B.S., M.S., and Ph.D. degrees in civil engineering from Chungnam National University, Korea in 2001, 2003, and 2008, respectively. His research interests are in the areas of GPS and geo-spatial information engineering. (jkpark@seoil.ac.kr) 